\title{
Importancia de la comunicación en la consulta veterinaria de pequeños animales: experiencia docente
}

\author{
Mercedes GARCÍA-SANCHO TÉLLEZ \\ Universidad Complutense de Madrid \\ mercgarc@vet.ucm.es \\ Alejandra VilLAescusa FeRnÁndeZ \\ Universidad Complutense de Madrid \\ alejandrav@vet.ucm.es \\ Fernando RodRÍGUEZ FRANCO \\ Universidad Complutense de Madrid \\ ferdiges@vet.ucm.es \\ Ángel SAINZ RoDRÍGUEZ \\ Universidad Complutense de Madrid \\ angelehr@vet.ucm.es
}

Recibido: $05 / 11 / 2012$

Aceptado: 23/01/2013

\begin{abstract}
Resumen
El actual plan de estudios de veterinaria se centra en las competencias técnicas sin hacer referencia a otro tipo de habilidades de tipo no técnico, como la empatía, el liderazgo o la comunicación eficaz. Dada la influencia de las habilidades comunicativas en el posterior desarrollo profesional, parece de vital importancia el fomento de estas enseñanzas entre los estudiantes de veterinaria. Por ello, realizamos este trabajo donde se facilitó la formación teórica y práctica al respecto a 45 estudiantes de veterinaria. Los resultados obtenidos por sí solos expresan la idoneidad y la necesidad de ampliar la formación de las competencias no técnicas relacionadas con las habilidades comunicativas entre los estudiantes de veterinaria.
\end{abstract}

Palabras clave: Comunicación, habilidades comunicativas, estudiantes, veterinaria

\section{Small Animals Veterinary Practice: the Importance of Communication Skills in Veterinary Students}

\begin{abstract}
The current veterinary curriculum emphasizes the importance of technical skills without addressing nontechnical skills such as empathy, leadership, and effective communication. Due to the known influence of communication skills within the professional development, teaching these communication skills to veterinary students could be of utmost importance. This paper presents a didactic and experiential training developed with 45 veterinary students of the Complutense University of Madrid. The results reinforce the importance of communication skills in veterinary medicine and the necessity of incorporate nontechnical competences into the curricula of veterinary schools.
\end{abstract}

Keywords: Communication, cummunication skills, students, veterinary medicine

\section{Referencia normalizada}

GARCÍA-SANCHO TÉLLEZ, Mercedes; VILLAESCUSA FERNÁNDEZ, Alejandra; RODRÍGUEZ FRANCO, Fernando; y SAINZ RODRÍGUEZ, Ángel (2013): "Importancia de la comunicación en la consulta veterinaria de pequeños animales: experiencia docente". Estudios sobre el Mensaje Periodístico. Vol. 19, Núm. especial marzo, págs.: 213-221. Madrid, Servicio de Publicaciones de la Universidad Complutense. 
Sumario: 1. Introducción. 2. Metodología. 3. Desarrollo. 4. Conclusiones. 5. Referencias bibliográficas.

\section{Introducción}

La importancia de la comunicación en medicina humana es un hecho. El reconocimiento de la necesidad de una enseñanza formal en relación a las habilidades comunicativas entre los alumnos de medicina es una realidad, habiéndose incorporado esta enseñanza en los planes de estudios de las facultades de medicina de diferentes lugares del mundo (Latham y Morris, 2007: 181). Los estudios realizados al respecto en medicina humana son muy numerosos. Así, desde hace 30 años se investigan las interacciones entre médico y paciente, habiéndose descrito su influencia sobre la salud del paciente, la satisfacción del paciente y del médico, el cumplimiento de las recomendaciones médicas y el riesgo de mala praxis (Roter y Frankel, 1992: 1097-1103; Shaw et al, 2004a: 678; Shaw, 2006: 386). En términos de comunicación, se ha descrito que las personas mostramos las mismas expectativas y necesidades en las relaciones que establecemos con profesionales tanto veterinarios como médicos (Anteyles, 1990: 1596). Por otra parte, cuando se pregunta a los propietarios sobre la relación con sus mascotas, hasta el $85 \%$ de los mismos consideran a sus animales como un miembro más de la familia (Brown y Silverman, 1999: 161). Parece por tanto probable, que la extrapolación de los resultados de medicina humana a la veterinaria clínica sea adecuada (Frankel, 2006: 20-27).

Históricamente, la formación de los veterinarios clínicos ha estado centrada en el aprendizaje de competencias técnicas, sin hacer referencia a otro tipo de habilidades de tipo no técnico, como la empatía, el liderazgo o la comunicación eficaz (Hafen et al ,2009: 429-430). Sin embargo, en la última década, la importancia de la comunicación en veterinaria es un tema de vigente actualidad, hecho respaldado por los múltiples estudios desarrollados al respecto (Hendrix et al, 2001; Radford et al, 2003; Lewis et al, 2003; Kogan et al, 2004; Latham y Morris, 2007; Chun et al, 2009; Cary et al, 2010). Así por ejemplo, el análisis de los factores implicados en la mala praxis veterinaria, revela que hasta en un $65 \%$ de los casos se relaciona con algún problema de comunicación, lo que indica que la formación en este campo es un componente importante (Cary et al, 2010: 130). Recientemente, ha surgido un creciente reconocimiento en la profesión veterinaria clínica de la importancia de este campo para un desarrollo profesional adecuado (Hafen et al, 2009: 429).

Dada la demostrada importancia de estas materias para el ejercicio veterinario, nos preguntamos, ¿se considera, entonces, adecuada la formación que reciben los alumnos al respecto? En esta línea, no se describen trabajos nacionales, y todos los estudios internacionales realizados, parecen coincidir en sus resultados, dejando ver que mientras que las habilidades clínicas, técnicas y científicas de la profesión veterinaria permanecen a un buen nivel, hay evidencia de que los veterinarios carecen de habilidades comunicativas y administrativas (Brown y Silverman, 1999: 161-183). Por ello, siendo conscientes de la importancia de la enseñanza de este tipo de competencias no técnicas, recientemente diversos centros universitarios de veterinaria a nivel mundial han incorporado en sus planes de estudio programas de formación específicos en este tipo de materias (Radford et al, 2003, Latham y Morris, 2007, Chun et al, 2009). 
No es este el caso, hasta el momento, de la Licenciatura ni del Grado en Veterinaria desarrollados en la Facultad de Veterinaria de la Universidad Complutense de Madrid. A lo largo de la Licenciatura, no se ve reflejada ninguna materia en relación a la comunicación, tanto oral como escrita, cuya importancia ya ha sido demostrada para los estudiantes de veterinaria. Entre las competencias generales de grado, sí aparece como primera "Ser capaz de expresarse correctamente en español, mostrando dominio del lenguaje técnico de su ámbito disciplinar", aunque sin embargo no se fomenta su consecución de manera concreta en ninguna materia.

En una encuesta realizada a 27 decanos de facultades de veterinaria americanas, los resultados se mostraron desesperanzadores, ya que en el 63\% de los casos se consideraba que los estudiantes de veterinaria no eran capaces de leer, escribir y hablar como deberían (Hendrix et al, 2001: 37). Sin la existencia de estudios específicos en las facultades españolas, y en concreto en la facultad de la UCM de Madrid, las sensaciones compartidas por el profesorado van en esa línea. Por ello, y dada la influencia de las habilidades comunicativas en el posterior desarrollo profesional, consideramos de vital importancia el fomento de estas enseñanzas destinadas a nuestros estudiantes de veterinaria. De esta manera, nació el trabajo que se presenta, dirigido a enriquecer la formación de los alumnos en temas de comunicación dentro del ámbito de una consulta veterinaria de pequeños animales.

\section{Metodología}

Para la llevar a cabo este trabajo, seleccionamos un grupo de 45 alumnos de los últimos cursos de la licenciatura de veterinaria de la Universidad Complutense de Madrid. Todos ellos, compartían la característica de ser alumnos internos de pequeños animales de Patología Médica y de la Nutrición. Los alumnos internos son estudiantes de últimos cursos que se incorporan a un departamento para participar en sus tareas, desempeñando labores compatibles con su dedicación al estudio, que contribuyan a su formación científica y profesional. Por ello, dada su condición de alumnos internos, su contacto con la realidad de la clínica veterinaria era amplio, debido a su participación a lo largo de todo el curso en el trabajo diario de las consultas del Hospital Clínico Veterinario Complutense. A estas consultas acuden un elevado número de propietarios de perros y gatos cuyas mascotas son atendidas en las diferentes especialidades en función de las patologías que presentan (Cardiología, Dermatología, Endocrinología y obesidad, Gastroenterología, Hematología, Hepatología, Medicina General, Neurología, Oftalmología y Oncología).

En primer lugar, los alumnos recibieron formación teórica multidisciplinar en forma de seminarios, resaltando los aspectos más importantes de la comunicación en el ámbito veterinario. Para ello, se contó con un equipo integrado por psicólogos clínicos y veterinarios clínicos con amplia experiencia en el campo. Los temas tratados hacían relación a la empatía, a técnicas de escucha activa, a la comunicación de malas noticias o a la eutanasia. Cada uno de los seminarios recibidos tuvo una duración aproximada de 2 horas, durante las cuales se fomentó la participación activa de los asistentes.

Posteriormente, una vez asimilados estos conceptos, se procedió a la aplicación práctica de los mismos a través de visualización y posterior debate de grabaciones de 
vídeo en las que se simulaban situaciones habituales vividas en la consulta de pequeños animales. En estas grabaciones, fueron veterinarios clínicos con formación previa en estos temas los que asumieron tanto el papel del veterinario como el del propietario. Estas sesiones tuvieron una duración aproximada de 2 horas.

Todos los participantes fueron invitados a completar unos cuestionarios en los que se valoró y puntuó una serie de aspectos relacionados con las actividades desarrolladas.

\section{Desarrollo}

De los 45 participantes, 8 eran hombres y 37 mujeres, todos ellos alumnos de $4^{\circ}$ o $5^{\circ}$ de la licenciatura de veterinaria. La elevada representación del sector femenino es un fiel reflejo del conjunto de estudiantes que integran la licenciatura de veterinaria, donde aproximadamente el $80 \%$ son mujeres.

A continuación, se exponen y se comentan los resultados derivados de la información vertida en los cuestionarios.

Un $90 \%$ consideraron que su formación previa al respecto era inexistente o muy escasa. El 98\% de los alumnos consideraron bastante o muy necesario llevar a cabo este tipo de actividades para completar su formación. En la licenciatura de veterinaria tal y como se imparte hasta el momento, no se incluyen asignaturas en las que se traten directamente temas relacionados con la comunicación, por lo que era de esperar este dato. Los alumnos, durante las prácticas clínicas desarrolladas en las consultas acuden a las mismas más centrados en aprender conocimientos técnicos que aquellos aspectos relacionados con la transmisión del mensaje.

El reconocimiento de la importancia de la comunicación en veterinaria ha sido responsable de la modificación de los programas docentes en muchas universidades, lográndose una incorporación progresiva de asignaturas al respecto. Así por ejemplo, a principios de los años 70, tan sólo una de las universidades americanas de veterinaria ofertaba un curso acerca de cómo hablar en público de poco más de una hora de duración (Terry, 1972: 405-415). Sin embargo, en la actualidad son ya muchas de ellas las que ofertan programas docentes en los que se incluyen asignaturas relacionada con temas de comunicación. Algo similar ha sucedido en Australia, donde la mayoría de las facultades de veterinaria en este continente ha incorporado la enseñanza de destreza o habilidades comunicativas a lo largo de los diferentes cursos de la carrera (Mills et al, 2009: web).

En Europa, la tendencia es similar. Reflejo de ello es la reciente creación con sede en la facultad de veterinaria de Londres (Royal Veterinary College) de un Centro de Excelencia en Enseñanza y Aprendizaje (Centre for Excellence in Teaching and Learning) que recibe el nombre de Lifelong Independent Veterinary Education (LIVE; www.live.ac.uk), del que cabe destacar el Clinical Skills Centre. Este último centro, está orientado a enseñar a los estudiantes de veterinaria la adquisición de diversas habilidades no técnicas de gran utilidad en la clínica.

A nivel nacional también se va evolucionando al respecto. Así por ejemplo, para el curso 2012/2013 la Facultad de Veterinaria de la Universidad de Córdoba incluye entre su oferta docente una asignatura optativa destinada a los alumnos de grado que lleva por nombre "Actitudes psicológicas del veterinario". 
Los temas tratados fueron considerados de elevado o muy elevado interés en el $98 \%$ de los casos. Como ya se ha comentado anteriormente, la ausencia de este tipo de contenidos en el programa docente actual incrementa el interés por los mismos.

El 93\% de los alumnos expresó que la formación recibida era de elevada o muy elevada utilidad para su futuro profesional. Como se ha expuesto con anterioridad, existe un gran déficit técnico y formativo en el campo de la comunicación de los profesionales veterinarios con sus clientes. En la mayoría de los casos, el método de aprendizaje se inicia cuando el alumno, ya licenciado, comienza a ejercer en el mundo profesional basándose en una técnica de ensayo y error. Es su propia experiencia la que le hace modificar sus actuaciones y dirigirlas en un sentido o en otro. Sin embargo, no siempre este método de aprendizaje es el más completo tal y como revela un estudio realizado con cerca de 100 veterinarios de 26 a 68 años de edad (Shaw JR et al, 2004b: 224-226). En dicho trabajo, la grabación en video de los profesionales durante la interacción con alguno de sus clientes permitió mostrar los siguientes datos: el 24\% de las grabaciones tenían un inicio brusco siendo el final brusco en el $12 \%$ de las mismas, en el $25 \%$ el veterinario no utiliza cuestiones abiertas, y tan sólo el $7 \%$ de los veterinarios se mostraron empáticos.

Sin embargo, es ampliamente conocida la importancia de la comunicación en la clínica veterinaria lo que se refleja en multitud de trabajos publicados al respecto. Se ha descrito que la mayoría de los propietarios dejarán de acudir a su veterinario si este muestra unas habilidades comunicativas y sociales pobres a la hora de atender a su mascota (Mills et al, 2009: web). Asimismo, cuando se plantea a los propietarios que definan los principales motivos por los que eligen a su veterinario son: que sea amable y con un carácter suave, y que sea respetuoso e informe de manera adecuada (Shaw JR et al, 2004a: 676). Parece por tanto que los propietarios valoran las habilidades no técnicas que muestran sus veterinarios.

El análisis de los factores implicados en la mala praxis veterinaria, revela que hasta en un $65 \%$ de los casos se relaciona con algún problema de comunicación (Cary et al, 2010: 130). Centrándonos en los recién graduados, se ha descrito que durante su primer año de trabajo hasta un $10 \%$ de los mismos reciben una queja contra ellos en relación a una pobre comunicación con el cliente (Radford et al, 2003: 422). Estos datos no hacen más que poner de manifiesto la necesidad de una comunicación propietarioveterinario adecuada, para que el veterinario pueda alcanzar y mantener un desarrollo profesional exitoso.

E1 91\% recomendaría intensamente a sus compañeros que asistieran a este tipo de actividades, solicitando además la realización de acciones similares en el 98\% de los casos. El carácter novedoso de la formación recibida, tanto por su contenido como por su forma, probablemente sea lo que motiva estas respuestas en los participantes. Hasta ahora se ha comentado la importancia del contenido, sin mencionar que los métodos que se empleen para desarrollar dichos contenidos tienen al menos la misma importancia. Estudios previos han puesto de manifiesto la gran utilidad de incorporar entre los métodos docentes el desarrollo de actividades en las que se simulan situaciones habituales vividas en una consulta (Radford et al, 2003, Chun et al, 2009). Se ha descrito que las simulaciones con clientes constituyen una herramienta educacional muy poderosa, siendo su- 
ficiente la realización de 2 ejercicios de simulación por alumno para que se observe un incremento significativo del grado de autoconfianza de estos alumnos (Mills et al, 2009: web). Además, mediante estas simulaciones se facilita que los alumnos descubran tanto sus puntos fuertes como sus debilidades (Mills et al, 2009).

Este tipo de actividades (role-play activities) se pueden llevar a cabo de diferentes formas, pudiendo ser representados los papeles de cliente o de veterinario por diferentes colectivos. Así por ejemplo, se han realizado estas actividades con estudiantes de veterinaria o de psicología (Chun et al, 2009: 197), e incluso con actores profesionales (Radford et al, 2003: 423-424). En nuestro caso, ambos papeles fueron interpretados por veterinarios cualificados. La elección de estos profesionales se basó en la amplia familiaridad de los mismos con las reacciones más habituales de veterinarios y de propietarios que se viven en una consulta de pequeños animales. En nuestra experiencia, la participación de veterinarios en estas actividades es muy adecuada. Sin embargo, para futuras actividades creemos que resultaría más enriquecedor para el aprendizaje de los alumnos su participación activa en estas actividades como protagonistas adquiriendo los diferentes roles descritos. Independientemente de la elección de los participantes de estas simulaciones, es importante la grabación de las mismas para su posterior valoración. La visualización de estas grabaciones permite su discusión en grupo favoreciendo una participación activa y dinámica de todos los asistentes. En un estudio previo, la visualización de este tipo de videos fue considerada una de las actividades más positivas realizadas por un grupo de alumnos, ya que les brindó la oportunidad de aprender observando la forma de comportarse de sus compañeros (Radford et al, 2003: 425). En esta línea, uno de los alumnos participantes nos transmitió que "Los videos son la mejor manera para transmitir los contenidos del seminario, incitando a la participación de los asistentes".

Finalmente, en el apartado destinado a la libre exposición de la opinión de los alumnos, estos fueron algunos de los comentarios que dejaron plasmados:

- "Que nos impartan este tema en alguna asignatura de la carrera"

- "Creo que el tema es imprescindible a tratar en la carrera"

- "Creo que esto debería impartirse en alguna asignatura de la propia carrera"

- "Debería impartirse en alguna asignatura para todos los alumnos"

- "Creo que este tipo de actividades son fundamentales para nuestro futuro como profesionales"

- "Siempre he dicho que la única asignatura que nos falta para cursar es la psicología y que es fundamental para entender a los propietarios. Por tanto, me parece una gran idea estos seminarios a los que acuden ponentes especializados en el tema y nos ayudan a mejorar como profesionales"

- "Me encantaría que se pudiesen dar más charlas formativas de este tipo"

Estos comentarios son un reflejo de los resultados comentados hasta ahora, donde los propios alumnos evidencian de manera más personal el déficit de formación que reciben en temas de comunicación, siendo conscientes de la importancia de la misma para su profesión. 


\section{Conclusiones}

Tal y como se ha hecho mención a lo largo del texto, el plan de estudios actual de la Licenciatura de Veterinaria se centra en las competencias técnicas, obteniendo como resultado alumnos muy cualificados con una amplia formación técnica. Sin embargo, los resultados presentados en este trabajo expresan por sí solos la idoneidad y la necesidad de ampliar la formación de las competencias no técnicas relacionadas con las habilidades comunicativas entre los estudiantes de veterinaria.

\section{Referencias bibliográficas}

ANTELYES, J. (1990): "Client hopes, client expectations". Journal of the American Veterinary Medical Association, 197. Illinois, American Veterinary Medical Association, pp.1596-1597.

BROWN JP, SILVERMAN JD (1999): "The current and future market for veterinarians and veterinary medical services in the United States". Journal of the American Veterinary Medical Association, 215. Illinois, American Veterinary Medical Association, pp. 161-183.

CARY JA, FARNSWORTH KD, KURTZ S (2010): “Telephone communication in emergency cases: a training program for veterinarian students". Journal of Veterinary Medical Education, 37. Washington, University of Toronto Press, pp. 130135.

CHUN R, SCHAEFER S, LOTTA CC, BANNING JA, SKOCHELAK SE (2009): "Didactic and experiential training to teach communication skills: the University of Wisconsin-Madison School of Veterinary Medicine collaborative experience". Journal of Veterinary Medical Education, 36. Washington, University of Toronto Press, pp. 196-201.

FRANKEL RM (2006): "Pets, vets and frets: what relationship-centered care research has to offer veterinary medicine". Journal of Veterinary Medical Education, 33. Washington, University of Toronto Press, pp. 20-27.

HAFEN M, RUSH BR, NELSON SC (2009): "Utilizing filmed authentic studentclient interactions as a communication teaching tool". Journal of Veterinary Medical Education, 36. Washington, University of Toronto Press, pp. 429-435.

HENDRIX CM, THOMPSON IK, MANN CJ (2001): “A survey of reading, writing, and oral communication skills in North American Veterinary Medical Colleges". Journal of Veterinary Medical Education, 28. Washington, University of Toronto Press, pp. 34-40.

KOGAN LR, BUTLER CL, LAGONI LK, BRANNAN JK, McCONNELL SM, HARVEY AM (2004): "Training in client relations and communication skills in veterinary medical curricula and usage after graduation". Journal of the American Veterinary Medical Association, 224. Illinois, American Veterinary Medical Association, pp. 504-507. 
LATHAM CE, MORRIS A (2007): "Effects of formal training in communication skills on the ability of veterinary students to communicate with clients". Veterinary Record, 160. Londres, British Veterinary Association, pp. 181-186.

LEWIS RE, KLAUSNER JS (2003): "Nontechnical competencies underlying career success as a veterinarian". Journal of the American Veterinary Medical Association, 222. Illinois, American Veterinary Medical Association, pp. 1690-1696.

MILLS J, BAGULEY J, COLEMAN G, MEEHAN M (2009): "Enhancing communication and life skills in veterinary students: Curriculum Development and Assessment of Methods". Disponible en: http://www.tlc.murdoch.edu.au/project /ecals/docs/ECALS_finalreport.pdf. [fecha de consulta: 17 de septiembre de 2012]

RADFORD AD, STOCKLEY P, TAYLOR IR, TURNER R, GASKELL CJ, KANEY S, HUMPHRIS G, MAGRATH C (2003): "Use of simulated clients in training veterinary undergraduates in communication skills". Veterinary Record, 152. Londres, British Veterinary Association, pp. 422-427.

ROTER D, FRANKEL R (1992): "Quantitative and qualitative approaches to the evaluation of the medical dialogue". Social Science \& Medicine, 34. Elsevier, pp. 10971103.

SHAW JR, ADAMS CL, BONNETT BN (2004a): "What can veterinarians learn from studies of physician-patient communication about veterinarian-client-patient communication?" Journal of the American Veterinary Medical Association, 224. Illinois, American Veterinary Medical Association, pp. 676-684.

SHAW JR, ADAMS CL, BONNETT BN, LARSON S, TOTER DL (2004b): "Use of the Roter interaction analysis system to analyze veterinarian-client-patient communication in companion animal practice". Journal of the American Veterinary Medical Association, 225. Illinois, American Veterinary Medical Association, pp. 222-229.

SHAW JR (2006): "Four core communication skills of highly effective practitioners". Veterinary Clinics of North America: Small Animal Practice, 36. Elsevier, pp. 385396.

TERRY LL (1972): "New horizons for veterinary medicine". Educación Médica y Salud, 6. Pp. 405-415. 


\section{Mercedes GARCÍA-SANCHO TÉLLEZ}

Universidad Complutense de Madrid. Facultad de Veterinaria Departamento de Medicina y Cirugía Animal Profesor Ayudante Doctor mercgarc@vet.ucm.es.

\section{Alejandra VILLAESCUSA FERNÁNDEZ}

Universidad Complutense de Madrid. Facultad de Veterinaria Departamento de Medicina y Cirugía Animal Profesor Ayudante Doctor alejandrav@vet.ucm.es

\section{Fernando RODRÍGUEZ FRANCO}

Universidad Complutense de Madrid. Facultad de Veterinaria Departamento de Medicina y Cirugía Animal

Profesor Titular de Universidad ferdiges@vet.ucm.es

\section{Ángel SAINZ RODRÍGUEZ}

Universidad Complutense de Madrid. Facultad de Veterinaria Departamento de Medicina y Cirugía Animal Profesor Titular de Universidad angelehr@vet.ucm.es 